\title{
Impact of preset and postset adaptive statistical iterative reconstruction- $V$ on image quality in nonenhanced abdominal- pelvic CT on wide-detector revolution CT
}

\author{
Zheng Zhu ${ }^{1 \#}$, Yanfeng Zhao ${ }^{1 \#}$, Xinming Zhao ${ }^{1}$, Xiaoyi Wang ${ }^{1}$, Weijun Yu ${ }^{1}$, Mancang Hu ${ }^{1}$, Xuan Zhang ${ }^{2}$, \\ Chunwu Zhou ${ }^{1}$ \\ ${ }^{1}$ Department of Diagnostic Radiology, National Cancer Center/National Clinical Research Center for Cancer/Cancer Hospital, Chinese Academy of \\ Medical Sciences and Peking Union Medical College, Beijing, China; ${ }^{2}$ GE Healthcare, Beijing, China
}

\#These authors contributed equally to this work.

Correspondence to: Xinming Zhao. Department of Diagnostic Radiology, National Cancer Center/National Clinical Research Center for Cancer/ Cancer Hospital, Chinese Academy of Medical Sciences and Peking Union Medical College, 17, Panjiayuan NanLi, Chaoyang District, Beijing 100021, China. Email: xinmingzh@sina.com.

Background: Adaptive statistical iterative reconstruction- $V$ technique (ASIR-V) is usually set at different strengths according to the different clinical requirements and scenarios encountered when setting scanning protocols, such as setting a more aggressive tube current reduction (defined as preset ASIR-V). Reconstruction with ASIR-V is useful after scanning using image algorithms to improve image quality (defined as postset ASIR-V). The aim of this study was to investigate the quality of images reconstructed with preset and postset ASIR-V, using the same noncontrast abdominal-pelvic computed tomography (CT) protocols in the same individual on a wide detector CT.

Methods: We prospectively enrolled 141 patients. The scan protocols in Groups A-E were 0\%, 20\%, 40\%, $60 \%$, and $80 \%$ preset ASIR-V, respectively, in the 256 wide-detector row Revolution CT (GE Healthcare, Waukesha, WI, USA). Each group was further divided into 5 subgroups with $0 \%, 20 \%, 40 \%, 60 \%$, and $80 \%$ postset ASIR-V, respectively. The 64-detector Discovery 750 HDCT (GE, USA) was used for Group F as a control group, using $0 \%, 20 \%, 40 \%, 60 \%$, and $80 \%$ ASIR, respectively. Image noise was measured in the spleen, aorta, and muscle. The CT attenuation and image noise were analyzed using the paired $t$-test; analysis of variance and post hoc multiple comparisons were made using the Student-Newman-Keuls (SNK) method.

Results: The CT attenuation in Groups A-F exhibited no significant difference between subgroups in three organs $(\mathrm{P}>0.05)$. Only with increasing preset ASIR-V\% (Groups A to E), did the image noise decrease,

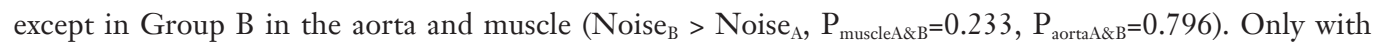
increasing postset ASIR-V or ASIR\% (Groups A and F), did the image noise decrease in the three organs. After preset and postset ASIR-V were combined, with preset ASIR-V\% being equal to postset ASIR-V\%, the image become similar to the corresponding preset ASIR-V part with the line of postset ASIR-V 0\% (baseline of each group). When preset ASIR-V\% was greater than the postset ASIR-V\%, the image noise was higher than the baseline of each group. When preset ASIR-V\% was less than the postset ASIR-V\%, the image noise was lower than the baseline of each group. The radiation dose from B to E decreased from $11.2 \%$ to $57.1 \%$. The CT dose index volume $\left(\mathrm{CTDI}_{\mathrm{vol}}\right)$ and dose length product (DLP) in Group F were significantly higher than those in Group A.

Conclusions: Using both preset and postset ASIR-V allows dose reduction, with a potential to improve image quality only when postset ASIR-V\% is higher than or equal to preset ASIR-V\%. The image quality depends on postset ASIR-V\%, whereas the decrease of radiation dose depends on preset ASIR-V\%. 
Keywords: Adaptive statistical iterative reconstruction-V technique (ASIR-V); wide-detector; computed tomography (CT); image noise; dose reduction

Submitted Nov 13, 2019. Accepted for publication Aug 24, 2020.

doi: $10.21037 /$ qims-19-945

View this article at: http://dx.doi.org/10.21037/qims-19-945

\section{Introduction}

The risk of radiation-induced carcinogenesis is a frequently raised concern (1-3), and is an issue particularly in patients with chronic medical conditions undergoing serial computed tomography (CT) examinations for disease management (4). There are many strategies for reducing radiation dose, including the use of tube current modulation, automatic exposure control, and automated kilovolt modulation $(5,6)$; nevertheless, all of these are associated with some compromise with image quality.

Adaptive statistical iterative reconstruction (ASIR), one of the most widely studied iterative reconstruction (IR) methods, reduces image noise and improves image quality; however, it cannot reduce radiation dose prospectively when scanning parameters remain unchanged $(7,8)$. As a hybrid IR algorithm, ASIR yields blended images between filtered back projection (FBP) and IR with IR percentages from $0 \%$ to $100 \%$, in which the percentage represents the contribution of the ASIR to the final images. Several studies have reported that ASIR results in quality issues such as artificial texture or blotchy appearance, particularly when high strength IR is used (9). Currently, ASIR is used to optimize image quality under the original scanning parameters and to reduce radiation dose while maintaining image quality by reducing the tube current. Nevertheless, there is no authorized standard for specific reduction of the tube current or voltage and the applied proportion of ASIR.

For these reasons, the latest version of a vendor-specific IR method, adaptive statistical iterative reconstruction-V (ASIR-V) (GE Healthcare, Waukesha, WI, USA), was developed. The ASIR-V series has the potential for prospective, clinically feasible dose reduction with better image quality than conventional ASIR, and shorter imaging processing time than model-based IR (MBIR). Compared to ASIR, which has object and system noise statistics modeling, ASIR-V also contains physics modeling. Compared with MBIR which has object, physics, system optics, and system noise statistics modeling, ASIR-V uses a less complex system model for forward projection that deemphasizes the system optics, resulting in a faster reconstruction time (10).
Some studies on phantom or in vivo have demonstrated the potential for an additional radiation dose reduction in the head, abdominopelvic, and coronary views using ASIR-V compared with ASIR or MBIR (11-15). In our study, we defined preset ASIR-V as ASIR-V that can influence the level of milliamperes $(\mathrm{mA})$ when using the automatic tube current modulation technique. The higher the preset ASIR-V strength, the lower the mA level. Meanwhile, postset ASIR-V reconstruction was defined as ASIR-V reconstruction after scanning. Nevertheless, to the best of our knowledge, there has been no in vivo study on the combined use of preset and postset ASIR-V for the abdominopelvic area in the same patient, in the same scan, and on the same wide-detector CT.

The purpose of this study was thus to prospectively determine whether CT images with preset ASIR-V plus postset ASIR-V might yield better quality images and decrease the radiation dose compared with CT images. This study was conducted by simply applying preset ASIR-V or/ and postset ASIR-V in the same patient, with non-contrast abdominal-pelvic CT scan on wide-detector Revolution CT.

\section{Methods}

\section{Clinical data}

This prospective random single-institution study was Health Insurance Portability and Accountability Actcompliant and was approved by our institutional review board (IRB) (NCC2016YZ-03). Written informed consent was provided by all patients. From March to May 2019, a total of 150 consecutive patients underwent noncontrast abdominal-pelvic CT scan for the evaluation of abdominal disease. The patients were randomly assigned to six groups. Exclusion criteria were pregnancy and age $<18$ years or $>80$ years. Nine patients were excluded due to having metallic foreign bodies. Finally, 141 patients (78 men and 63 women, age $21-78$ years; mean age, $56 \pm 11$ years) were enrolled in this study \{Groups A [22], B [24], C [25], D [24], $\mathrm{E}$ [24], and F [22]\}. Participants' height and weight were recorded before the CT scan was performed. 


\section{CT scanning technique}

Groups A to E underwent 256-wide-detector row abdominal-pelvic CT (Revolution CT; GE Healthcare, Milwaukee, WI, USA). The common scan parameters were as follows: $120 \mathrm{kVp}$ tube voltage; automatic tube current modulation system (Auto-mAs, GE Healthcare) with a noise index (NI) of 9 for a $5 \mathrm{~mm}$ slice thickness, slice interval $5 \mathrm{~mm}$; field of view (FOV) $35 \mathrm{~cm}$; matrix $512 \times 512$; and detector pitch 0.984 . When scanning protocols were being set, the selected strength of ASIR-V varied according to different clinical requirements and scenarios (defined as preset ASIR-V for dose reduction and image quality setting in our study). Reconstruction with ASIR-V could be performed after scanning by using image algorithms (defined as postset ASIR-V for a $5 \mathrm{~mm}$ slice thickness and slice interval soft image reconstruction in our study).

The scan protocols were $0 \%, 20 \%, 40 \%, 60 \%$, and $80 \%$ preset ASIR-V, respectively. The raw data of Groups A-E were reconstructed with different proportions of postset ASIR-V $(0 \%, 20 \%, 40 \%, 60 \%$, and $80 \%$, respectively). As a control, Group F underwent 64-detector row abdominal-pelvic CT (Discovery CT750 High Definition; GE Healthcare, Milwaukee, WI, USA) with no ASIR-V. The raw data of Group F were reconstructed with different proportions of ASIR $(0 \%, 20 \%, 40 \%$, $60 \%$, and $80 \%$, respectively). The scan range started at the diaphragm and scanned through to the pubic symphysis.

Image noise of both preset ASIR-V $=0 \%$ and postset ASIR-V $=0 \%$ was used as a general baseline to evaluate image quality. Additionally, when the preset ASIR-V equaled $0 \%$, the image quality of each group (A-E) was considered to be the baseline value of each group. All image sets were then placed into a research folder on a picture archiving and communication system (PACS) diagnostic workstation (CareStream, Carestream Health, Inc, Onex, Toronto, ON, Canada).

\section{Qualitative and quantitative analysis}

Qualitative image analysis was performed by two independent blinded radiologists with 18 and 15 years of experience in abdominal CT (Y. Z. and Z.Z.). Reviewers initially received standardized instructions and were trained using the image sets from four patients not included in this study. Images were presented to reviewers with a window width of 400 Hounsfield units (HU) and window level of 40
$\mathrm{HU}$; however, reviewers could vary the window width/level at will. No time limits were placed on the image review process. All data were randomized, rendered anonymous, and reviewed on a PACS diagnostic workstation (CareStream, Carestream Health, Inc, Onex, Toronto, ON, Canada) for assessment of subjective quality. Image quality was evaluated with a scale ranging from 1 to 5 (worst-best), as previously reported. The scoring was defined as follows: grading point 1: poor, impaired image quality limited by excessive noise; 2 : adequate, reduced image quality with either poor vessel wall definition or excessive image noise; 3: good, minimal effect of image noise and limitation of a low contrast resolution; 4: very good, good attenuation of vessel lumen and delineation of vessel walls, with relatively minimal image noise; 5: excellent, clear delineation of vessel walls, with limited perceived image noise. The mean value of Likert scores from the two observers was used for analysis.

Quantitative analysis was performed by a radiologist with 15 years of experience in abdominal imaging, through the placement of circular regions of interest (ROIs) in three areas: (I) the spleen, (II) the abdominal aorta (above the celiac artery), and (III) muscle. The CT number (HU) and image noise [defined as the acquired standard deviation (SD) for CT numbers] were recorded. The ROIs were placed on the erector spinae muscle distant from artifacts or bones, and were two-thirds the size of the vessels (ROI $=150-200 \mathrm{~mm}^{2}$ ) for measuring the aorta; another ROI of at least $400 \mathrm{~mm}^{2}$ was placed in the spleen in each series. Each spleen ROI was carefully placed in a separate, relatively homogeneous area of the spleen away from the discernible vessels or focal changes in attenuation. The measurements were repeated three times, and the average values were calculated. The volume CT dose index $\left(\mathrm{CTDI}_{\mathrm{vo}}, \mathrm{mGy}\right)$ and dose length product (DLP, $\mathrm{mGy} \cdot \mathrm{cm}$ ) were recorded with reference to the dose report (16). The accuracy of CTDI $_{\mathrm{vol}}$ and DLP was regularly examined as part of the quality control program at our institution.

Interobserver variability between the two radiologists assessing subjective image quality of Gourps A-E was estimated using kappa statistics. The scale included the following intervals: $<0.20$, poor; $0.21-0.40$, fair; $0.41-0.60$, moderate; $0.61-0.80$, substantial; and $0.81-1.00$, almost perfect $(17,18)$.

\section{Statistical analysis}

Attenuation, image noise, and effective dose measurements 
of CT were analyzed using the paired $t$-test and oneway analysis of variance (ANOVA), and post hoc multiple comparisons were made using the Student-Newman-Keuls (SNK) method. A P value $<0.05$ was considered to indicate a significant difference. All data were analyzed using SPSS software version 13.0 (IBM, Chicago, IL, USA).

\section{Results}

\section{Clinical data and CT attenuation}

Differences in age and body mass index (BMI) amongst the six groups were not statistically significant $(\mathrm{P}>0.05)$ (Table 1$)$. For all three organs, CT attenuation in Groups A-F exhibited no significant difference between the subgroups $(\mathrm{P}>0.05)$.

\section{Preset ASIR-V}

For the spleen (Table 2), the image noise in each group decreased only with the increased percentage of preset ASIR-V ( $0 \%, 20 \%, 40 \%, 60 \%$, and 80\%) from Groups AE (Figure 1, red line).

In the aorta (Table 3, Figure 1, green line) and muscle (Table 4, Figure 1, blue line), after percentages of the preset ASIR-V in Groups A-E were increased, the image noise in each group was lower than the previous ones, except in Group B $\left(\right.$ Noise $_{B}>$ Noise $\left._{A}, P_{\text {muscleA\&B } B}=0.233, P_{\text {aortaA\&B }}=0.796\right)$.

\section{Postset ASIR-V (Control group-ASIR)}

Image noise of the spleen (Table 2, Figure 2A), aorta (Table 3, Figure 2B), and muscle (Table 4, Figure 2C) in groups A and $\mathrm{F}$ decreased from subgroup postset ASIR-V $0 \%$ in $\mathrm{A} /(\mathrm{ASIR} 0 \%$ in $\mathrm{F})$ to postset ASIR-V $80 \%$ in $\mathrm{A} /(\mathrm{ASIR}$ $80 \%$ in $\mathrm{F}$ ), and the differences between each subgroup in the same group were significant, except for subgroups ASIR 0\% and ASIR 20\% in Group F for all three organs $\left(\mathrm{P}_{\text {spleen-Asir0\%\&Asir } 20 \%}=0.260, \mathrm{P}_{\text {aorta-Asir0\% \&Asir20\% }}=0.209, \mathrm{P}_{\text {muscle- }}\right.$ Asir0\% \&Asir20\% $=0.072$ ).

\section{Preset ASIR-V and postset ASIR-V}

With fixed preset ASIR-V (within Groups B-E) in all three organs, the image noise had a gradual downward trend when the percentage of postset ASIR- $\mathrm{V}$ was increased (subgroups 20-80\%) (Figure 3A,B,C).

When the percentage of preset ASIR- $V$ was higher than the percentage of postset ASIR-V, the image noise was significantly higher than the group baseline $(\mathrm{P}<0.05)$. In contrast, when the percentage of preset ASIR- $V$ was lower than the percentage of postset ASIR-V, the image noise was significantly lower than the group baseline $(\mathrm{P}<0.05)$. When the percentage of preset ASIR- $V$ was equal to the percentage of postset ASIR- $\mathrm{V}$, the image quality was equal to or slightly lower than when the percentage of preset ASIR-V and postset ASIR-V were both $0 \%$, and the image noise was similar to the corresponding preset ASIR-V part with the line of postset ASIR-V $0 \%$ [0\% and 20\% postset ASIR-V in Group B $\left(\mathrm{t}_{\text {spleen }}=-1.916, \mathrm{P}_{\text {spleen }}=0.068 ; \mathrm{t}_{\text {aorta }}=-0.673\right.$, $\left.\mathrm{P}_{\text {aorta }}=0.508 ; \mathrm{t}_{\text {muscle }}=0.937, \mathrm{P}_{\text {muscle }}=0.359\right), 0 \%$ and $40 \%$ postset ASIR-V in Group C $\left(\mathrm{t}_{\text {spleen }}=-1.962, \mathrm{P}_{\text {spleen }}=0.061 ; \mathrm{t}_{\text {aorta }}=0.528\right.$, $\left.\mathrm{P}_{\text {aorta }}=0.603 ; \mathrm{t}_{\text {muscle }}=0.599, \mathrm{P}_{\text {muscle }}=0.555\right), 0 \%$ and $60 \%$ postset ASIR-V in Group D ( $\mathrm{t}_{\text {spleen }}=0.084, \mathrm{P}_{\text {spleen }}=0.933 ; \mathrm{t}_{\text {aorta }}=-1.071$, $\left.\mathrm{P}_{\text {aorta }}=0.295 ; \mathrm{t}_{\text {muscle }}=-0.482, \mathrm{P}_{\text {muscle }}=0.634\right), 0 \%$ and $80 \%$ postset ASIR-V in Group $\mathrm{E}\left(\mathrm{t}_{\text {spleen }}=-1.021, \mathrm{P}_{\text {spleen }}=0.318\right.$; $\left.\left.\mathrm{t}_{\text {aorta }}=1.714, \mathrm{P}_{\text {aorta }}=0.100 ; \mathrm{t}_{\text {muscle }}=1.910, \mathrm{P}_{\text {muscle }}=0.069\right)\right]$ (Tables 1-4).

\section{Radiation dose}

According to the manufacturer's data, the radiation dose from Groups B-E decreased from $11.2 \%$ to $57.1 \%$ (Table 1). The radiation dose in Groups B-D decreased significantly $(\mathrm{P}<0.001)$. The radiation dose of Group B was lower than that of Group A; however, the difference was not significant $(\mathrm{P}>0.05)$. The radiation dose of Group $\mathrm{E}$ was lower than that of Group D; however, the difference was not significant $(\mathrm{P}>0.05)$.

The CTDIvol and DLP in Group F were significantly higher than those of Group $\mathrm{A}(\mathrm{P}<0.001)$ (Table 1).

\section{Subjective evaluation and kappa}

All image quality scores of Groups A-E were $\geq 3$ (good) with respect to overall image quality of the abdominal organs (Figure $4 A, B, C$ ). There was an almost perfect interobserver agreement with respect to image quality for each group

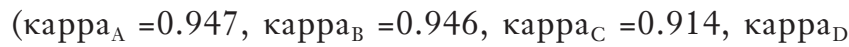
$=0.931$, Kappa $\left._{\mathrm{E}}=1.000\right)($ Table 5).

\section{Discussion}

To the best of our knowledge, the findings of this study have not been reported in previous studies. We found that 
Table 1 The clinical data and scan dose in different groups

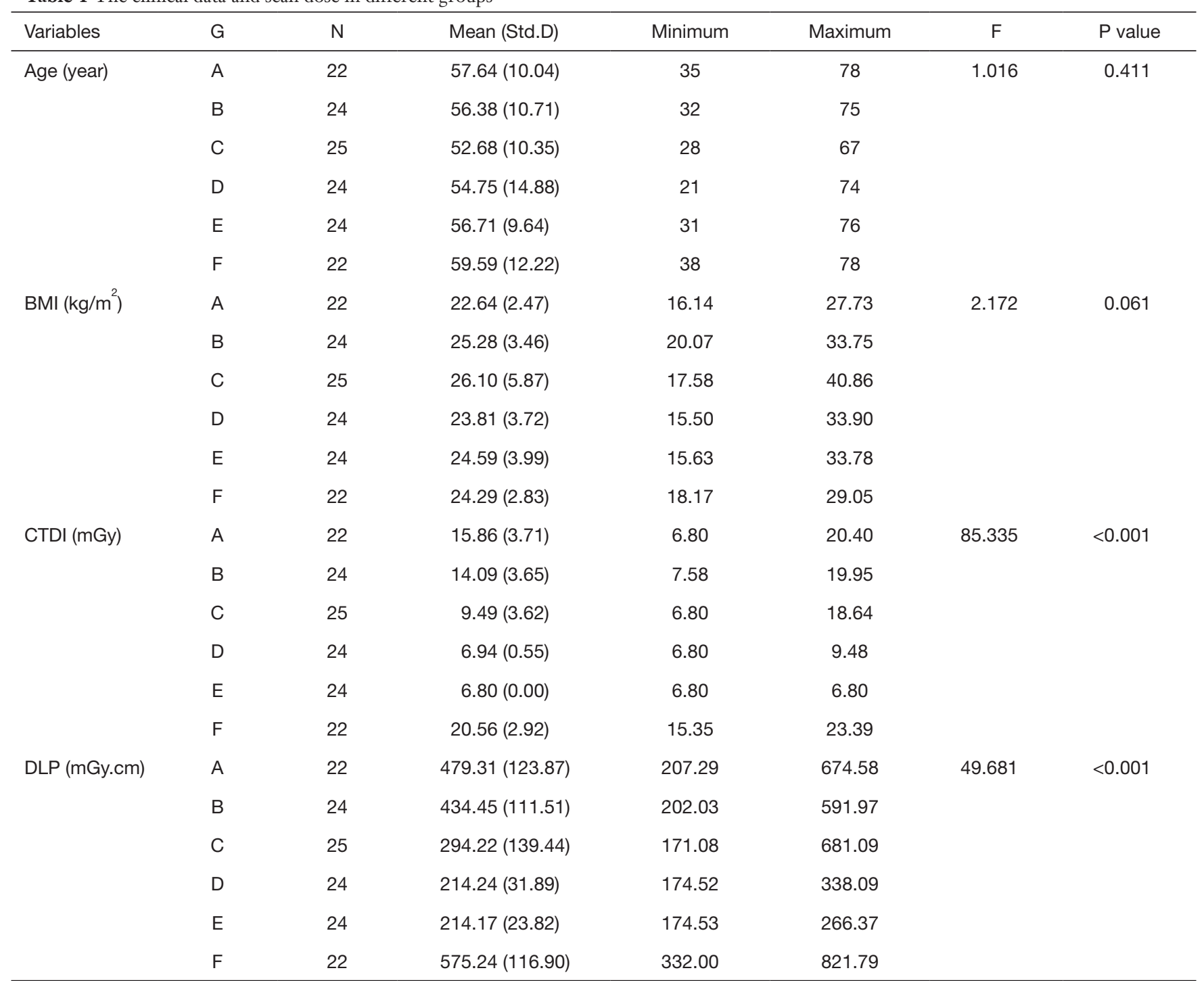

G, group; N, number; BMI, body index mass (calculated as weight divided by square of height; $\mathrm{kg} / \mathrm{m}^{2}$ ); Std.D, Std. deviation; CTDI, CT dose index; DLP, dose length product.

when the percentage of postset ASIR-V was higher than the percentage of preset ASIR-V, the image quality was better than the group baseline (postset ASIR-V $=0 \%$ ), and the greater the difference between the two percentages, the greater the image quality improvement, suggesting the image noise decreased accordingly.

Improving CT image quality and radiation dose reduction remain important areas of research. This has led to the optimization of CT imaging protocols, with radiation doses as low as reasonably achievable without compromising diagnostic image quality (19). Most studies have been based on the application of automatic mAs and automatic $\mathrm{KV}$ technology, with further use of various algorithms to achieve this purpose. In the present study, we investigated three modes in experiment groups (Groups A-E): the simple application of preset ASIR-V, simple application of postset ASIR-V, and the combined application of preset ASIR-V with postset ASIR-V. We included an ASIR group as a control group (Group F). After comparison, we found that simply applying preset ASIR-V (a dose reduction method which lowers the tube current) or postset ASIR-V (an IR algorithm) is beneficial for the image quality of non- 
Table 2 Image noise of spleen in six groups and five subgroups

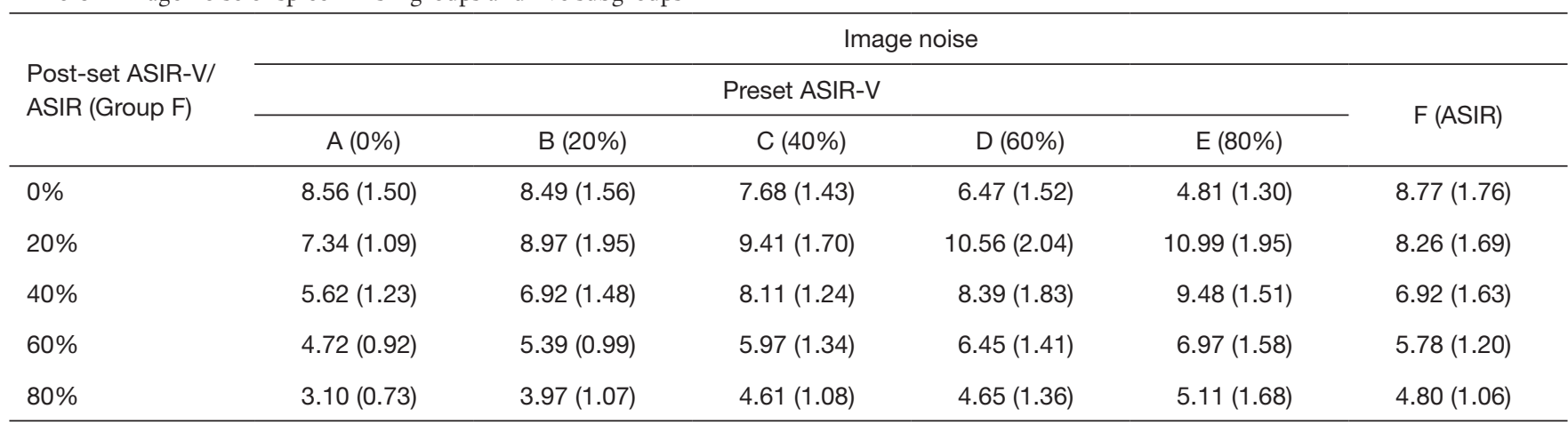

Groups A-E use different percentage of preset and postset ASIR-V and Group F only use ASIR reconstruction. The data of image noise was represented by mean (Std.D).

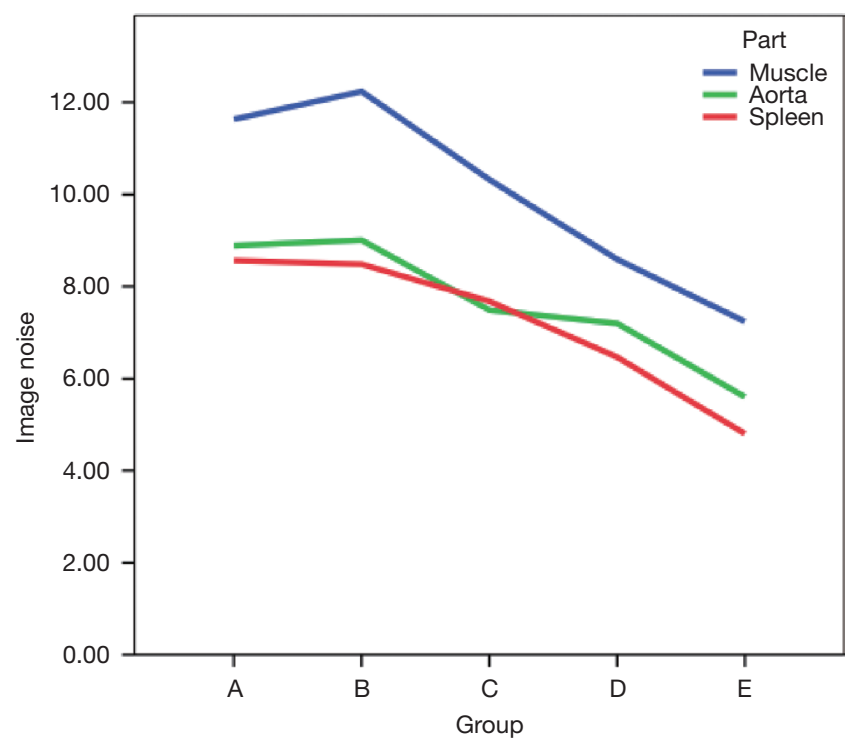

Figure 1 Line chart of image noise of different percentages of preset ASIR-V in three different organs. Group A $=0 \%$ preset ASIR-V, Group B $=20 \%$ preset ASIR-V, Group C $=40 \%$ preset ASIR-V, Group D $=60 \%$ preset ASIR-V, Group E $=80 \%$ preset ASIR-V. ASIR-V, adaptive statistical iterative reconstruction-V technique.

contrast abdominal-pelvic CT scanning. When applying both, the image quality showed no further improvement, except when the percentage of postset ASIR- $V$ was equal to or higher than the percentage of preset ASIR-V. Image quality depends on the percentage of postset ASIR-V, and the decrease in radiation dose depends upon the percentage of preset ASIR-V. In other words, the system likely expects that users would use corresponding ASIR-V postset to reconstruct the ASIR-V preset acquisition.

Similar results were obtained when applying postset ASIR-V or ASIR alone (Groups A and F). In other words, when the percentage of postset ASIR-V or ASIR increased, image noise decreased and the image quality increased. This is consistent with the results of previous studies $(8,20,21)$. When applying ASIR or postset ASIR-V with the unchanged original automatic mAs technology (i.e., NI fixation) and KV fixation, image quality may improve; however, radiation dose does not decrease. The data in Groups A and F were expected to be the same; in fact, the image noise in A was lower than that of F except for muscle and aorta in the subgroup postset ASIR-V 0\% (ASIR 0\%). The participants were randomly selected, and there were no significant differences with respect to age, BMI, or other characteristics. One reason could be that the two CT scanners had different detector widths, or perhaps ASIR-V is the improved version of ASIR. The Discovery $750 \mathrm{HD}$ has a 4-cm detector width, while the Revolution CT has an $8-\mathrm{cm}$ width. The image noise of detectors invariably increases with width, which results in lower image quality.

Postset ASIR-V can only increase image quality, not reduce radiation dose, yet preset ASIR-V can prospectively reduce radiation dose without decreasing image quality $(9,22)$, which provides simultaneous radiation dose reduction and ensures similar or unchanged image quality. A certain percentage of preset ASIR-V is used to compensate for the decrease in image quality caused by reduced radiation dose. In Groups B, C, D, and E, 20\%, 40\%, 60\%, and $80 \%$ preset ASIR-V were used, respectively. With the simple application of preset ASIR-V, when the percentage increased, the image quality remained unchanged or 
Table 3 Image noise of aorta in six groups and five subgroups

\begin{tabular}{|c|c|c|c|c|c|c|}
\hline \multirow{3}{*}{$\begin{array}{l}\text { Post-set ASIR-V/ } \\
\text { ASIR (Group F) }\end{array}$} & \multicolumn{6}{|c|}{ Image noise } \\
\hline & \multicolumn{5}{|c|}{ Preset ASIR-V } & \multirow{2}{*}{$\mathrm{F}(\mathrm{ASIR})$} \\
\hline & $\mathrm{A}(0 \%)$ & B (20\%) & C (40\%) & D (60\%) & $E(80 \%)$ & \\
\hline $0 \%$ & $8.89(1.42)$ & $9.01(1.60)$ & 7.49 (1.51) & $7.20(1.91)$ & $5.61(1.29)$ & $8.68(1.53)$ \\
\hline $20 \%$ & $7.48(1.25)$ & $9.09(1.67)$ & $9.15(2.03)$ & $11.43(2.72)$ & $11.13(2.13)$ & $8.22(1.39)$ \\
\hline $60 \%$ & $4.87(1.14)$ & $5.59(0.88)$ & 5.94 (1.39) & $7.36(1.97)$ & $7.19(1.41)$ & $6.12(1.04)$ \\
\hline $80 \%$ & $3.89(1.26)$ & $4.26(0.77)$ & $4.31(1.23)$ & $5.47(1.75)$ & $5.36(0.96)$ & $5.23(0.78)$ \\
\hline
\end{tabular}

Groups A-E use different percentage of preset and postset ASIR-V and Group F only use ASIR reconstruction. The data of image noise was represented by mean (Std.D).

Table 4 Image noise of muscle in six groups and five subgroups

\begin{tabular}{|c|c|c|c|c|c|c|}
\hline \multirow{3}{*}{$\begin{array}{l}\text { Post-set ASIR-V/ } \\
\text { ASIR (Group F) }\end{array}$} & \multicolumn{6}{|c|}{ Image noise } \\
\hline & \multicolumn{5}{|c|}{ Preset ASIR-V } & \multirow{2}{*}{$F(A S I R)$} \\
\hline & $\mathrm{A}(0 \%)$ & B (20\%) & C (40\%) & D (60\%) & $E(80 \%)$ & \\
\hline $0 \%$ & $11.63(1.27)$ & 12.24 (1.92) & $10.32(2.15)$ & $8.59(1.70)$ & 7.24 (1.52) & $10.52(1.51)$ \\
\hline $20 \%$ & $9.75(1.45)$ & 12.05 (1.73) & $12.65(2.50)$ & $13.28(2.59)$ & $14.19(2.48)$ & $9.79(1.45)$ \\
\hline $60 \%$ & $6.47(1.08)$ & $7.92(1.25)$ & 8.19 (1.69) & 8.69 (1.59) & $9.53(1.58)$ & $7.18(1.16)$ \\
\hline $80 \%$ & $5.04(1.15)$ & $5.89(1.37)$ & $5.94(1.36)$ & $6.39(1.29)$ & $6.87(1.35)$ & $6.06(1.05)$ \\
\hline
\end{tabular}

Groups A-E use different percentage of preset and postset ASIR-V and Group F only use ASIR reconstruction. The data of image noise was represented by mean (Std.D).

increased slightly; however, the radiation dose decreased gradually (from $11.2 \%$ to $57.1 \%$ ). This result is similar to those reported previously (23-25).

Postset ASIR-V improves the image quality of CT but cannot reduce the radiation dose prospectively. Preset ASIR- $V$ can reduce the radiation dose, but the improvement of image quality is not substantial (26). Therefore, we attempted to combine preset ASIR- $V$ and postset ASIR-V for the same patients in the same scan to evaluate the image quality and radiation dose. To the best of our knowledge, this has not been reported in previous studies. We found that when the percentage of postset ASIR-V was higher than the percentage of preset ASIR-V, image quality was better than the baseline of each group (the corresponding preset ASIR-V part with the line of postset ASIR-V 0\%), and when the difference between the two percentages was greater, image quality improved even more, suggesting that image noise decreased. Contrastingly, when the percentage of postset ASIR-V was lower than the percentage of preset ASIR-V, the image quality was lower than the baseline of each group and decreased even more when the difference between the two percentages was greater. Reductions in image quality correlated with increased image noise. When the percentage of postset ASIR-V was equal to the percentage of preset ASIR-V, the image quality (noise) was similar to the baseline of each group. In short, we found that the image quality was not further improved by the combination of postset ASIR-V and preset ASIR-V. When the percentages of postset ASIR-V and preset ASIR-V were set simultaneously, the effect of superimposition of this hybrid algorithm was not achieved; rather, there was only relay on either the percentage of postset ASIR-V or preset ASIR-V. The percentage of postset ASIR-V was the final percentage of the FBP + ASIR hybrid algorithm when both were used at the same time. However, the percentage of preset ASIR- $\mathrm{V}$ does not play any role in 

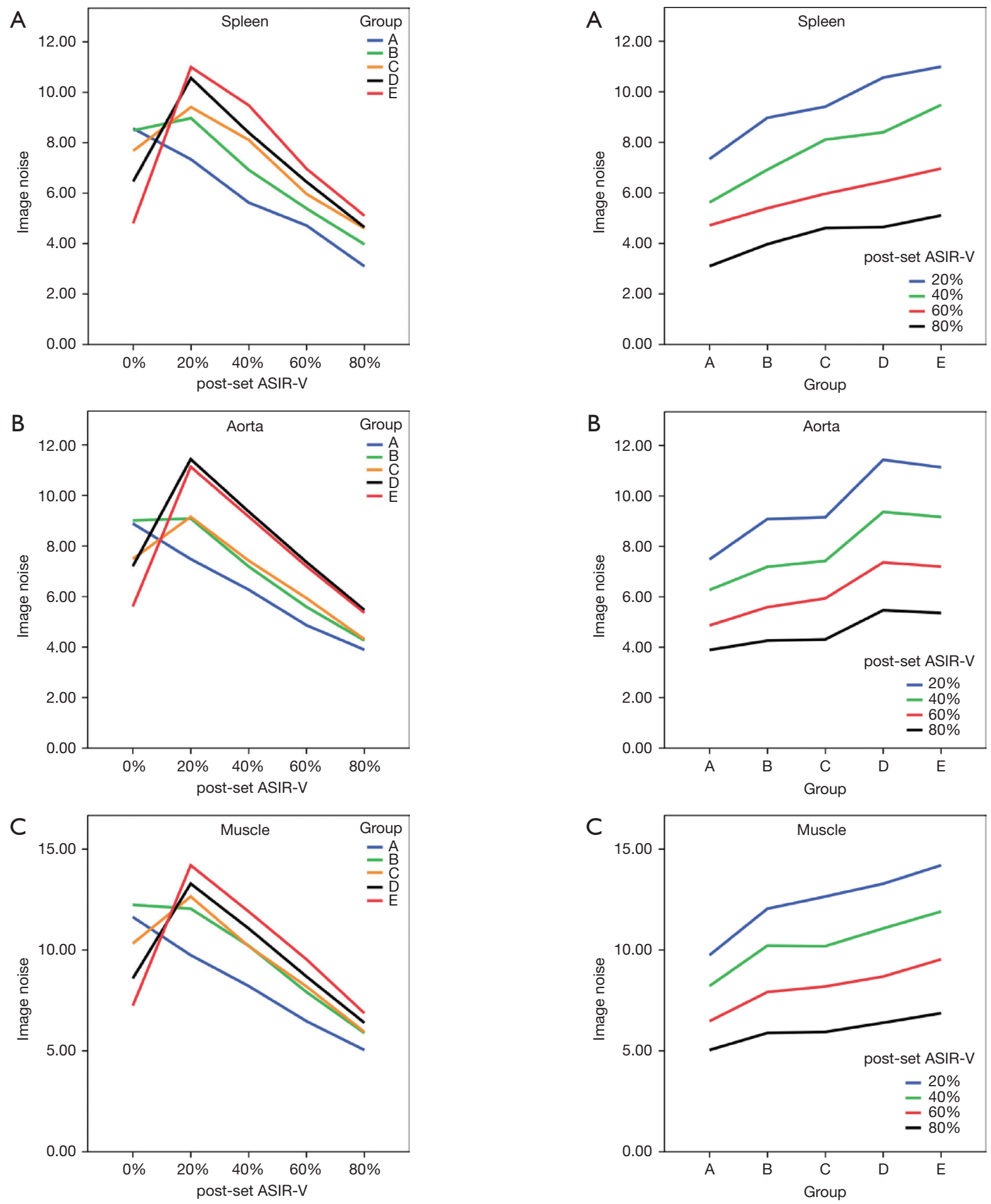

Figure 2 Line chart of image noise of different preset ASIR-V group in subgroup postset ASIR-V 0\%, 20\%, 40\%, 60\%, and 80\% of the spleen, aorta, and muscle. (A) The line chart of the image noise of the spleen. (B) The line chart of the image noise of the aorta. (C) The line chart of the image noise of muscle. ASIR-V, adaptive statistical iterative reconstruction- $\mathrm{V}$ technique.

Figure 3 Line chart of the image noise of postset ASIR-V 20\%, $40 \%, 60 \%$, and $80 \%$ in different preset ASIR-V values of the spleen, aorta, and muscle. (A) The line chart of image noise of the spleen. (B) The line chart of the image noise of the aorta. (C) The line chart of the image noise of muscle. ASIR-V, adaptive statistical iterative reconstruction- $\mathrm{V}$ technique. 

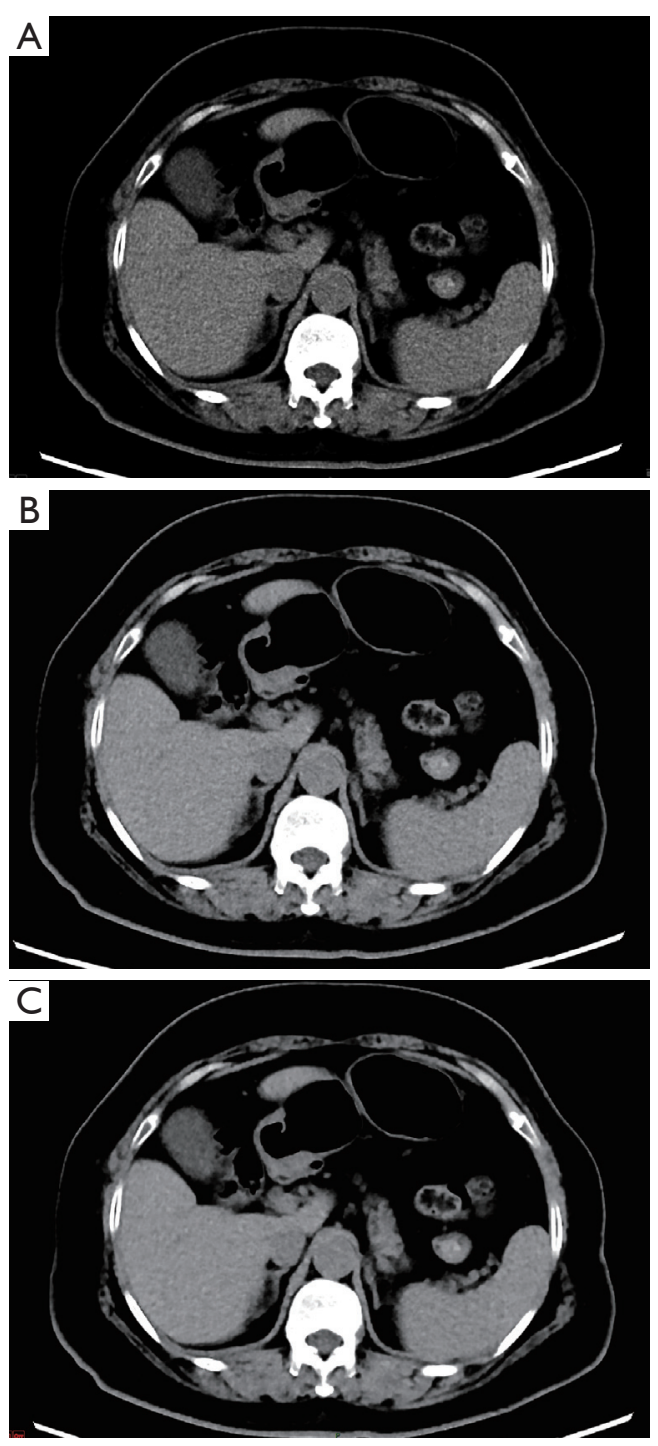

Figure 4 Subjective evaluation of image quality in the abdominal organs in preset ASIR-V 80\% with different percentages of postset ASIR-V. (A) The image quality scores were 3 (good) in preset ASIR-V 80\% with postset ASIR-V 20\%. (B) The image quality scores were 4 (very good) in the same patients with preset ASIR-V $80 \%$ and postset ASIR-V 60\%. (C) The image quality scores were 5 (excellent) in the same patient with preset ASIR-V $80 \%$ and postset ASIR-V 80\%. ASIR-V, adaptive statistical iterative reconstruction- $\mathrm{V}$ technique.

image reconstruction; that is, the effect of preset ASIR-V was not clearly shown. The decreased radiation dose was automatically reduced by the initial percentage of preset ASIR-V. This also explains why image quality was worse than the baseline of each group when the percentage of preset ASIR-V was higher than that of postset ASIR-V, and the image quality was worse when the difference between the two percentages was larger. Even when the percentage of preset ASIR-V was higher, the radiation dose was automatically lowered in a corresponding proportion in order to compensate for image quality loss with the related amount of preset ASIR-V. Actually, postset ASIR-V that only adds a small percentage does not compensate for all the loss of image quality due to its radiation dose reduction; therefore, the radiation dose decreases and the image quality decreases accordingly. So, the expectation from this system would be that operators would use the corresponding ASIR-V postset to reconstruct the ASIR-V preset acquisition.

The radiation dose in Groups B, C, and D decreased significantly, while there were no significant differences between Groups A and B, D and E. The reason for this may be related to the scanning protocol. We applied automatic mAs technology; however, we simultaneously and manually set the range of mAs to be $200-600 \mathrm{mAs}$, to maintain image quality for clinical diagnosis. Therefore, mAs was restricted from automatically rising or falling to reach the borderline of 10 or $740 \mathrm{mAs}$ as originally intended with the CT scanner. Groups A and B were closer to $600 \mathrm{mAs}$, while Groups D and E were closer to $200 \mathrm{mAs}$, resulting in no particular change in overall radiation dose.

There were several limitations to our study. Firstly, only three relatively homogenous organs (the spleen, aorta, and muscle) were included, while heterogeneous organs such as the liver or pancreas were not considered. Secondly, image noise was the only image quality metric used in our study. It is now well known that the IR algorithms are very good at suppressing image noise but can alter image texture. We did not evaluate lesion detectability or localization in keeping with other previous studies. Thirdly, only five percentage intervals of preset ASIR-V were selected, while other studies have used divisions such as $12.5 \%, 25 \%, 37.5 \%, 50 \%$, $75 \%$, and $100 \%$ (27), or every $10 \%$ from $0 \%$ to $100 \%(24)$. Future inclusion of an increased number of groups, such as every $10 \%$ from $0 \%$ to $100 \%$ would improve representation and help to further determine the applicability of our results to those percentages.

In conclusion, simply applying either preset ASIR-V or postset ASIR-V is beneficial to image quality of noncontrast abdominal-pelvic CT scanning. When applying both, the image quality showed no further improvement, except when the percentage of postset ASIR-V was equal or higher than the percentage of preset ASIR-V. Image quality depends 
Table 5 The subjective evaluation in different percentage of preset ASIR-V

\begin{tabular}{|c|c|c|c|c|c|c|}
\hline Radiologist 1 & Category & \multicolumn{5}{|c|}{ Radiologist 2} \\
\hline \multirow[t]{3}{*}{ Group A } & Good & 75 & 1 & 0 & 0.947 & $<0.001$ \\
\hline & Very good & 3 & 55 & 0 & & \\
\hline & Excellent & 0 & 0 & 7 & & \\
\hline \multirow{2}{*}{ Group B } & Very good & 1 & 50 & 0 & & \\
\hline & Excellent & 0 & 0 & 7 & & \\
\hline \multirow[t]{2}{*}{ Group C } & Good & 2 & 0 & 0 & 0.914 & $<0.001$ \\
\hline & Very good & 0 & 35 & 2 & & \\
\hline \multirow{2}{*}{ Group D } & Very good & 1 & 124 & 0 & & \\
\hline & Excellent & 0 & 1 & 11 & & \\
\hline \multirow[t]{3}{*}{ Group E } & Good & 140 & 0 & 0 & 1.000 & 0.007 \\
\hline & Very good & 0 & 1 & 0 & & \\
\hline & Excellent & 0 & 0 & 0 & & \\
\hline
\end{tabular}

on the percentage of postset ASIR-V, and the decrease in radiation dose depends upon the percentage of preset ASIR-V.

\section{Acknowledgments}

We would like to acknowledge Lin Li (Department of Diagnostic Radiology, National Cancer Center/National Clinical Research Center for Cancer/Cancer Hospital, Chinese Academy of Medical Sciences and Peking Union Medical College), Jianying Li (GE Healthcare), and Shuai Zhang (GE Healthcare) for their contributions in this work. Funding: This study was funded by the Fundamental Research Funds for the Central Universities (3332018080).

\section{Footnote}

Conflicts of Interest: All authors have completed the ICMJE uniform disclosure form (available at http://dx.doi. org/10.21037/qims-19-945). The authors have no conflicts of interest to declare.

Ethical Statement: This prospective random single-institution study was Health Insurance Portability and Accountability Act-compliant, was approved by our institutional review board (NCC2016YZ-03), and written informed consent was provided by participants for the publication of this study and any accompanying images. A copy of the written consent is available for review by the Editor-in Chief of this journal.

Open Access Statement: This is an Open Access article distributed in accordance with the Creative Commons Attribution-NonCommercial-NoDerivs 4.0 International License (CC BY-NC-ND 4.0), which permits the noncommercial replication and distribution of the article with the strict proviso that no changes or edits are made and the original work is properly cited (including links to both the formal publication through the relevant DOI and the license). See: https://creativecommons.org/licenses/by-nc-nd/4.0/.

\section{References}

1. Brenner DJ, Hall EJ. Computed tomography--an increasing source of radiation exposure. $\mathrm{N}$ Engl J Med 2007;357:2277-84. 
2. Pearce MS, Salotti JA, Little MP, McHugh K, Lee C, Kim KP, Howe NL, Ronckers CM, Rajaraman P, Sir Craft AW, Parker L, Berrington de Gonzalez A. Radiation exposure from CT scans in childhood and subsequent risk of leukaemia and brain tumours: a retrospective cohort study. Lancet 2012;380:499-505.

3. Dahal S, Budoff MJ. Low-dose ionizing radiation and cancer risk: not so easy to tell. Quant Imaging Med Surg 2019;9:2023-6.

4. Sodickson A, Baeyens PF, Andriole KP, Prevedello LM, Nawfel RD, Hanson R, Khorasani R. Recurrent CT, cumulative radiation exposure, and associated radiationinduced cancer risks from CT of adults. Radiology 2009;251:175-84.

5. Chang W, Lee JM, Lee K, Yoon JH, Yu MH, Han JK, Choi BI. Assessment of a model-based, iterative reconstruction algorithm (MBIR) regarding image quality and dose reduction in liver computed tomography. Invest Radiol 2013;48:598-606.

6. Karmazyn B, Liang Y, Klahr P, Jennings SG. Effect of tube voltage on CT noise levels in different phantom sizes. AJR Am J Roentgenol 2013;200:1001-5.

7. Pickhardt PJ, Lubner MG, Kim DH, Tang J, Ruma JA, del Rio AM, Chen GH. Abdominal CT with modelbased iterative reconstruction (MBIR): initial results of a prospective trial comparing ultralow-dose with standarddose imaging. AJR Am J Roentgenol 2012;199:1266-74.

8. Zhu Z, Zhao XM, Zhao YF, Wang XY, Zhou CW.

Feasibility Study of Using Gemstone Spectral Imaging (GSI) and Adaptive Statistical Iterative Reconstruction (ASIR) for Reducing Radiation and Iodine Contrast Dose in Abdominal CT Patients with High BMI Values. PLoS One 2015;10:e0129201.

9. Lim K, Kwon H, Cho J, Oh J, Yoon S, Kang M, Ha D, Lee J, Kang E. Initial phantom study comparing image quality in computed tomography using adaptive statistical iterative reconstruction and new adaptive statistical iterative reconstruction v. J Comput Assist Tomogr 2015;39:443-8.

10. Fan J, Yue M, Melnyk R. Benefits of ASiR-V reconstruction for reducing patient radiation dose and preserving diagnostic quality in CT exams. (white paper) Waukesha, WI: GE Healthcare, 2014.

11. Goodenberger MH, Wagner-Bartak NA, Gupta S, Liu X, Yap RQ, Sun J, Tamm EP, Jensen CT. Computed Tomography Image Quality Evaluation of a New Iterative Reconstruction Algorithm in the Abdomen (Adaptive Statistical Iterative Reconstruction-V) a Comparison With
Model-Based Iterative Reconstruction, Adaptive Statistical Iterative Reconstruction, and Filtered Back Projection Reconstructions. J Comput Assist Tomogr 2018;42:184-90.

12. Benz DC, Grani C, Mikulicic F, Vontobel J, Fuchs TA, Possner M, Clerc OF, Stehli J, Gaemperli O, Pazhenkottil AP, Buechel RR, Kaufmann PA. Adaptive Statistical Iterative Reconstruction-V: Impact on Image Quality in Ultralow-Dose Coronary Computed Tomography Angiography. J Comput Assist Tomogr 2016;40:958-63.

13. De Marco P, Origgi D. New adaptive statistical iterative reconstruction ASiR-V: Assessment of noise performance in comparison to ASiR. J Appl Clin Med Phys 2018;19:275-86.

14. Kim HG, Lee HJ, Lee SK, Kim HJ, Kim MJ. Head CT: Image quality improvement with ASIR-V using a reduced radiation dose protocol for children. Eur Radiol 2017;27:3609-17.

15. Park C, Choo KS, Kim JH, Nam KJ, Lee JW, Kim JY. Image Quality and Radiation Dose in CT Venography Using Model-Based Iterative Reconstruction at $80 \mathrm{kVp}$ versus Adaptive Statistical Iterative Reconstruction-V at 70 kVp. Korean J Radiol 2019;20:1167-75.

16. Maldjian PD, Goldman AR. Reducing radiation dose in body CT: a primer on dose metrics and key CT technical parameters. AJR Am J Roentgenol 2013;200:741-7.

17. Landis JR, Koch GG. The measurement of observer agreement for categorical data. Biometrics 1977;33:159-74.

18. Svanholm H, Starklint H, Gundersen HJ, Fabricius J, Barlebo H, Olsen S. Reproducibility of histomorphologic diagnoses with special reference to the kappa statistic. APMIS 1989;97:689-98.

19. ICRP Publication 105. Radiation protection in medicine. Ann ICRP 2007;37:1-63.

20. Brady SL, Moore BM, Yee BS, Kaufman RA. Pediatric CT: implementation of ASIR for substantial radiation dose reduction while maintaining pre-ASIR image noise. Radiology 2014;270:223-31.

21. Hara AK, Paden RG, Silva AC, Kujak JL, Lawder HJ, Pavlicek W. Iterative reconstruction technique for reducing body radiation dose at CT: feasibility study. AJR Am J Roentgenol 2009;193:764-71.

22. Gatti M, Marchisio F, Fronda M, Rampado O, Faletti R, Bergamasco L, Ropolo R, Fonio P. Adaptive Statistical Iterative Reconstruction-V Versus Adaptive Statistical Iterative Reconstruction: Impact on Dose Reduction and Image Quality in Body Computed Tomography. J Comput Assist Tomogr 2018;42:191-6.

23. Chen LH, Jin C, Li JY, Wang GL, Jia YJ, Duan HF, Pan N, 
Guo J. Image quality comparison of two adaptive statistical iterative reconstruction (ASiR, ASiR-V) algorithms and filtered back projection in routine liver CT. Br J Radiol 2018;91:20170655.

24. Tang H, Yu N, Jia Y, Yu Y, Duan H, Han D, Ma G, Ren $\mathrm{C}, \mathrm{He}$ T. Assessment of noise reduction potential and image quality improvement of a new generation adaptive statistical iterative reconstruction (ASIR-V) in chest CT. Br J Radiol 2018;91:20170521.

25. Lee S, Kwon H, Cho J. The Detection of Focal Liver Lesions Using Abdominal CT: A Comparison of Image Quality Between Adaptive Statistical Iterative Reconstruction V and Adaptive Statistical Iterative

Cite this article as: Zhu Z, Zhao Y, Zhao X, Wang X, Yu W, Hu $M$, Zhang $X$, Zhou C. Impact of preset and postset adaptive statistical iterative reconstruction- $\mathrm{V}$ on image quality in nonenhanced abdominal-pelvic CT on wide-detector revolution CT. Quant Imaging Med Surg 2021;11(1):264-275. doi: 10.21037/ qims-19-945
Reconstruction. Acad Radiol 2016;23:1532-8.

26. Kwon H, Cho J, Oh J, Kim D, Kim S, Lee S, Lee J. The adaptive statistical iterative reconstruction- $\mathrm{V}$ technique for radiation dose reduction in abdominal CT: comparison with the adaptive statistical iterative reconstruction technique. Br J Radiol 2015;88:20150463.

27. Solomon J, Marin D, Roy Choudhury K, Patel B, Samei E. Effect of Radiation Dose Reduction and Reconstruction Algorithm on Image Noise, Contrast, Resolution, and Detectability of Subtle Hypoattenuating Liver Lesions at Multidetector CT: Filtered Back Projection versus a Commercial Model-based Iterative Reconstruction Algorithm. Radiology 2017;284:777-87. 